\title{
Instrument for Exotic Particles Search in Cosmic Rays (INSTEPS)
}

\section{George Bashindzhagyan*}

M.V. Lomonosov Moscow State University (RU)

E-mail: george.bashindzhagyandcern.ch

\section{Ephraim Fischbach}

Purdue University (IN, USA)

\section{Gagik Hovsepyan}

Yerevan Physics Institute (AM)

\section{Natalia Korotkova}

M.V. Lomonosov Moscow State University (RU)

\section{Nikolai Sinev}

University of Oregon (OR, USA)

\begin{abstract}
An Instrument for an exotic particle search (INSTEPS) in cosmic rays is proposed for detailed investigation of cosmic ray flux in the charge region 0.5 - 2 minimum-ionizing particles (mip), where a few strange results have been registered. These results could not be understood because of poor charge resolution. There is also $0.1-0.5 \mathrm{mip}$ region, which is practically unknown because of a limited signal to noise ratio. The instrument includes a set of self-triggered modules with 6 layers of $1 \mathrm{~mm}$ thick silicon microstrip detectors $10 \times 10 \mathrm{~cm}^{2}$ size for precise charge measurements. Every module can be also used as a separate device in many other experiments where precise charge determination is required. For exotic particle search a group of the charge measuring modules is placed on the top of a scintillator calorimeter. The calorimeter will measure particle energy and trajectory and generate a special trigger for the charge modules when the energy is above $50 \mathrm{GeV}$. This serves to decrease particle flux, because the already mentioned anomalies have been observed between high energy hadrons. We plan to expose the instrument at mountain level where it can register the particles that have arrived from space as well as those generated in the atmosphere.
\end{abstract}

35th International Cosmic Ray Conference - ICRC2017

10-20 July, 2017

Bexco, Busan, Korea

${ }^{*}$ Speaker. 


\section{Introduction}

To identify rare exotic particles among a huge flux of well-known ones, two major parameters are usually measured: a particle charge and energy. The common and not expensive method of energy measuring is a calorimeter and for a particle charge determination $\mathrm{dE} / \mathrm{dX}$ measuring works rather well. But a serious problem appears if we want to measure a charge precisely. So called Landau fluctuations of ionization have a typical half width about $35-45 \%$ for silicon and $70-100 \%$ for gas detectors. It's also asymmetric with a long tail, which makes it even more difficult to separate particles with higher charge. The situation becomes much worse if an exotic particle flux is very low. A serious improvement can be achieved if a few layers of detectors are used to measure the same particle $\mathrm{dE} / \mathrm{dX}$.

Figure 1 shows the difference in particle separation with a single layer of typical silicon pad detector (left plot) and with a system of six layers of such detectors (right plot). For simulation $200 \mathrm{GeV}$ protons have been mixed with $4 \%$ of $2 \mathrm{e} / 3$ quarks, $4 \%$ of $4 \mathrm{e} / 3$ diquarks and $25 \%$ of alpha particles. One can see that not only $2 \mathrm{e} / 3$ but also $4 \mathrm{e} / 3$ will be well separated with six layers of silicon. A special Rank statistic method has been used for 6 layers data analysis, because determining a regular mean value practically does not help due to a long tail of Landau fluctuations.
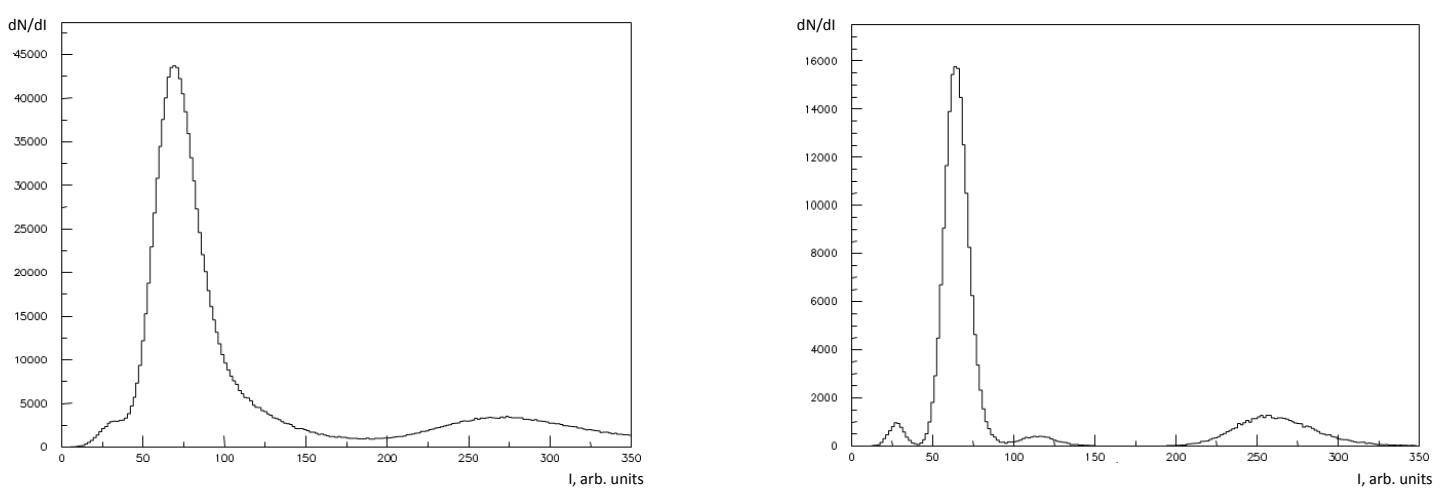

Figure 1: Ionization spectrum for $200 \mathrm{GeV}$ protons $(100 \%)$ with admixture of alpha particles (25\%) and particles with charge $2 / 3$ and $4 / 3$ e (4\% and $4 \%$ ). Signal to noise ratio is 10 . Left plot: one layer of silicon detectors is used. Right plot: six layers are used.

There are a few experiments where strange effects have been observed but could not be carefully analyzed because of pure charge measuring. The proposed INSTEPS device will help to check the unusual results presented below, and to observe carefully the region of charges less than 1e which was practically unknown because a limited signal to noise ratio. $1 \mathrm{~mm}$ thick layers of silicon strip detector instead of typical 0.3-0.4 mm allow us to obtain about 3 times higher output signal together with 3 times less input capacitance, and to measure a particle charge down to 0.1e.

\section{Aragats experiment}

This experiment [1] is unusual because 24 layers of gas proportional counter (PC) for precise charge measurements have been combined with hadron calorimeter and a hodoscope (Fig. 2). The experiment was performed at the Aragats cosmic ray station (Armenia) at altitude of $3200 \mathrm{~m}$ above 


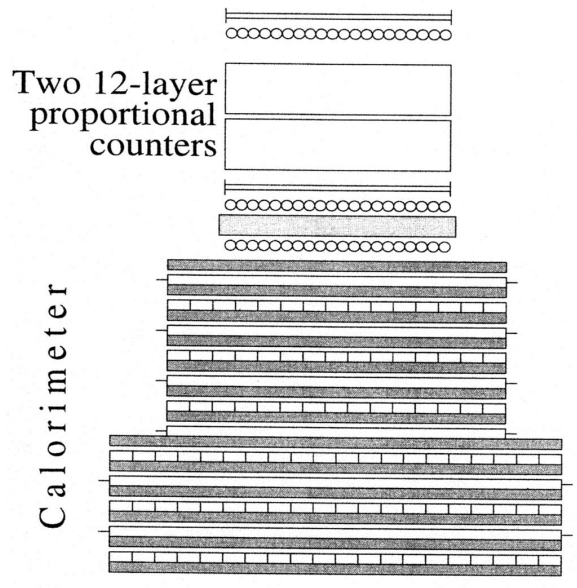

Figure 2: The structure of Aragats experiment.
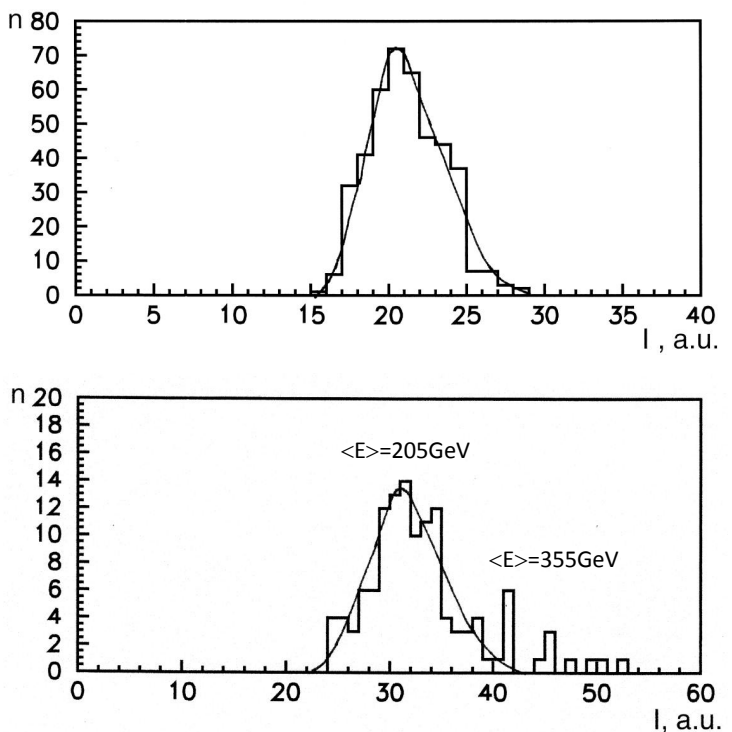

Figure 3: Top: ionization spectrum of $0.6 \mathrm{GeV}$ muon used for calibration. Bottom: ionization spectrum of hadrons. 14 events with ionization outside superimposed calibration curve have much higher average energy.

sea-level. The system was triggered by the calorimeter if hadron energy was above $80 \mathrm{GeV}$. Only single hadron type particles without accompanying air shower have been analyzed. $0.6 \mathrm{GeV}$ muons have been also registered for proportional counter calibration and for keeping PC gas amplification stable.

Two plots in Fig. 3 show muon and hadron ionization distributions. One can see that hadrons create 1.5 times higher ionization because of Relativistic Rise of Ionization (RRI) in gases. A calibration curve obtained by fitting the muon distribution has been used in the analysis of the hadron events and to separate a group of events, which had a considerably higher ionization. The hadrons inside the calibration curve are a mixture of protons and pions. It means that they are on the plateau of the RRI curve and none of single charged hadrons may have higher ionization. But 14 events above this level have been observed. The probability that this is a random deviation is $5 \times 10^{-4}$. We cannot exactly evaluate their charge because it depends on their mass according to the RRI.

If, for example, we suppose that they are $4 \mathrm{e} / 3$ diquarks their mass could be estimated as 30 $\mathrm{GeV}$. But the most interesting and intriguing fact is that the average energy of these 14 events is $355 \mathrm{GeV}$ while it is only $205 \mathrm{GeV}$ for regular single charged hadrons. The probability that this is a random deviation is less than 0.01 .

\section{ATIC-2 single charge particle ionization analysis}

The Advanced Thin Ionization Calorimeter (ATIC) [2] was designed to measure the composition and energy spectra of $Z=1$ to 28 cosmic rays over the energy range $10 \mathrm{GeV}$ to $100 \mathrm{TeV}$. 


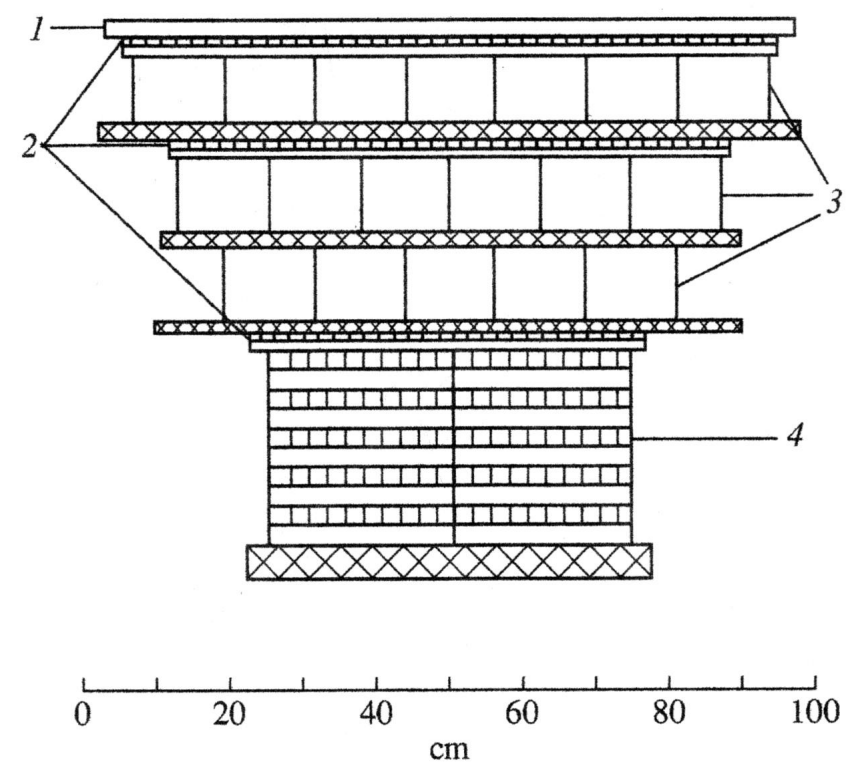

Figure 4: The layout of the ATIC device: (1) silicon matrix; (2) scintillator detectors; (3) carbon target; and (4) BGO ionization calorimeter.

The structure of ATIC device is shown in Fig. 4. The first time it was launched as a long duration balloon test flight on December 28, 2000 from McMurdo, Antarctica. The second science flight of ATIC started on December 29, 2002 also from McMurdo and was completed on January 18, 2003 [3]. We tried to analyze the small region of the charge (ionization) distribution from zero to alpha particles. Unfortunately only one layer of silicon pad detectors was used in ATIC silicon matrix. This seriously limits the possibility to separate particles with a charge less than 1e, and makes it practically impossible particles with a charge 1-1,5e separation.

Fig. 5 shows a histogram of ionization distribution near the proton peak for all the particles with energy deposited in the BGO calorimeter above $50 \mathrm{GeV}$. The right smooth curve fitted to the proton peak is a normalized ATIC silicon matrix detector response to 1 mip particle. Left smooth curve is a distribution of the events, which do not belong to the proton distribution. One can see that the relative half width of both curves is approximately identical (50\%). The maximum on the right curve corresponds to the most probable ionization of the regular protons ( 0.87 arb. units). It agrees with the maximum of alpha particle ionization position (not shown), which is 4 times higher because of the 2 times higher charge. The maximum of the left curve with "additional" events corresponds to ionization of 0.46 arb. units or 0.53 of proton ionization. If we suppose that the "additional" events are real particles then their charge can be estimated as $0.73 \mathrm{e}$. The right plot on Fig. 5 presents the same ionization distribution but for only central $60 \%$ of the silicon matrix area. One can see that the fraction of "additional" events relative to proton one is less there.

Fig. 6 presents an angular distribution for the protons (above $50 \mathrm{GeV}$ ) (the left plot) and for the "additional" events (the right plot). One can see that the "additional" events angular distribution has a visible maximum around $35^{\circ}$. 

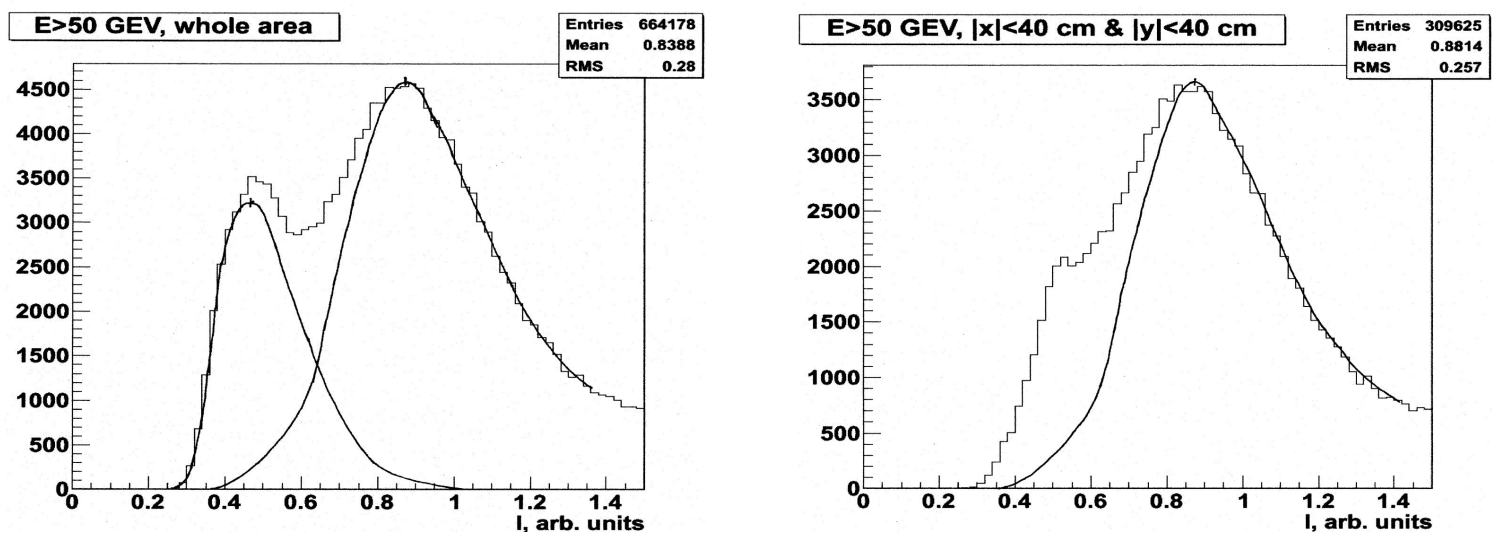

Figure 5: The ionization spectra for charged particles with energy $\mathrm{E}>50 \mathrm{GeV}$ measured during the ATIC second flight. Left: whole area of the silicon matrix is used for analysis. Right: only central part of the silicon matrix ( $60 \%$ ) in use.
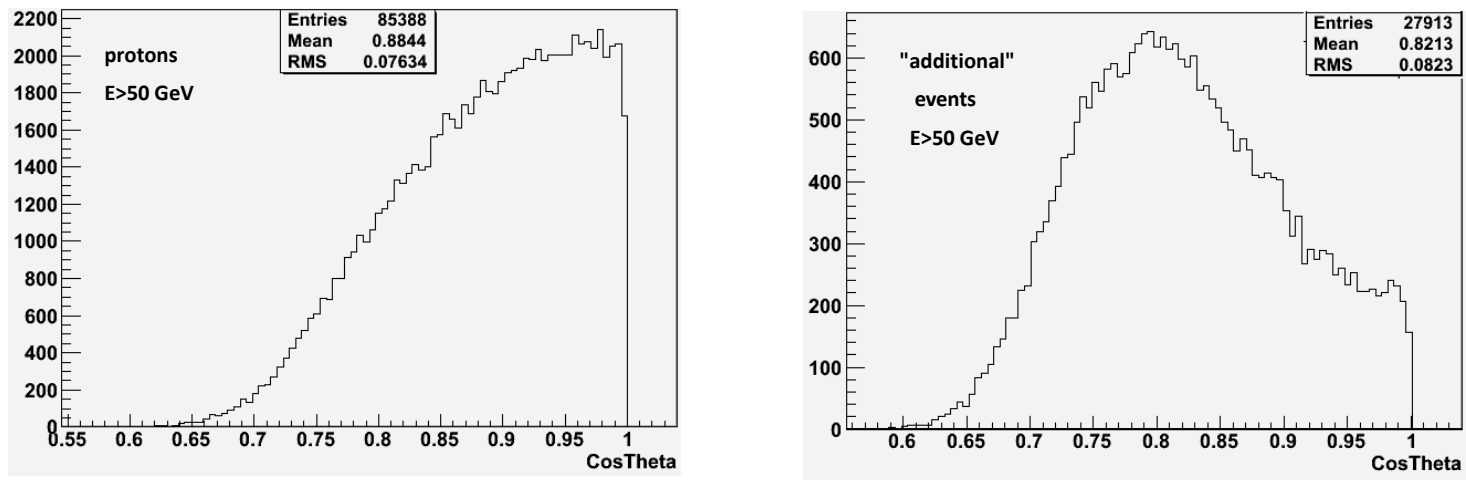

Figure 6: Angular distributions for protons and "additional" events.

\section{The INSTEPS device}

It is assumed that the device has to consist of two main parts: A hadron calorimeter to measure a particle energy and a system of silicon detectors to measure precisely a particle charge. At the same time we plan to make it as cheap as possible but using the modern technologies. We propose to use relatively thin (about $25 \mathrm{X}_{0}$ ) but fast hadron scintillating calorimeter about $75 \mathrm{x} 75 \mathrm{~cm}^{2}$ size (Fig.7). It has to generate a trigger when the energy is above $50 \mathrm{GeV}$ (can be adjusted) and to determine hadron cascade position and direction, however can also be triggered by the charge measuring system.

The use of a six layer silicon system needs serious discussion. There is a traditional approach to build large area detectors using relatively small separate silicon detectors with complete overlapping to avoid gaps. This seems to be really important for accelerator experiments, but creates a lot of problems with mechanical support, cooling, many interconnections etc.

In our cosmic ray experiment we do not need to measure a charge of every hadron which hits the calorimeter. We can make a system of charge measuring modules. Every module can be much smaller than the calorimeter but will be triggered by the calorimeter. If a hadron misses all the charge modules we only lose a certain per cent of information. A smaller number of modules just 
requires longer exposure.

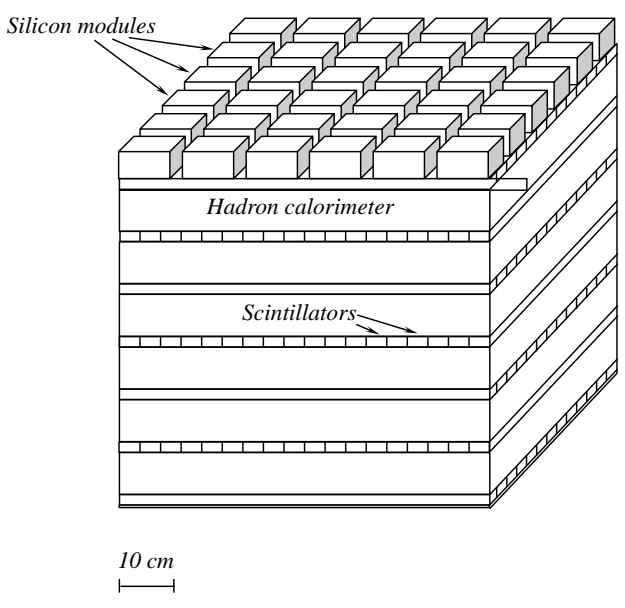

Figure 7: The device schematic view.
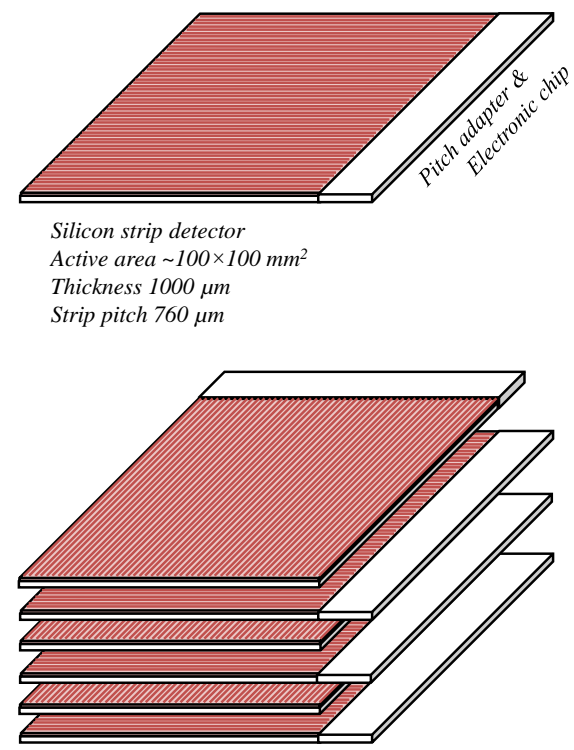

Charge measuring module

Figure 8: Charge measuring module.

Each charge measuring module has its own electronics and can also work as a completely independent self-triggered device. This means that in addition to high energy hadrons (when triggered by calorimeter) it can register all the cosmic ray particles including exotic ones. It also can be used in many other experiments were precise charge measurements are required. The serious advantage of such an approach is a possibility to combine the calorimeter with only one or a few modules at the beginning as a cheapest version of the setup and to increase the number of modules step by step depending on the obtained results.

The next point is to make the module as simple as possible. We propose to use single silicon strip detectors about $10 \times 10 \mathrm{~cm}^{2}$ size and $1 \mathrm{~mm}$ thick to improve signal to noise ratio. With 6 layers of the silicon the module can be 5-6 cm thick and about $12 \times 12 \mathrm{~cm}^{2}$ size (Fig. 8). In this case the number of modules in use can be from 1 and up to 36 for that size of the calorimeter. To minimize the cost of the experiment, and to simplify maintaining the device we do not keep in mind a balloon or satellite experiment but plan to expose the setup at mountain level at the same Aragats Cosmic Ray Station in Armenia where we have worked many years ago (3200 m above sea level). Excellent infrastructure, nice and friendly people, will help us to keep the experiment under control and collect the data continuously. Hadron flux will be essentially lower there but exposure time is unlimited. One more advantage is a possibility to register particles arrived from space as well as those generated in the atmosphere.

\section{Conclusion}

The proposed rather simple and cheap experiment is not only one more attempt to search for 
exotic particles in cosmic rays. It will help to check and clarify many strange results obtained during many years of searches for exotic particles, and especially fractionally charged particles searches, which could not be proved or rejected with limited charge resolution devices.

\section{References}

[1] G. L. Bashindzhagyan, L. I. Sarycheva, N. B. Sinev, Search for new particles among the single cosmic ray hadrons, in proceedings of the 16th International Cosmic Ray Conference (1979), vol. 6, p. 143.

[2] J. H. Adams Jr. for the ATIC collaboration, Preliminary results from the first flight of ATIC: The silicon matrix, in proceedings of the 27th International Cosmic Ray Conference (2001), p. 2127.

[3] J. P. Wefel, J. H. Adams, H. S. Ahn, G. L. Bashindzhagyan, K. E. Batkov et al., The ATIC Science Flight in 2002-03: Description and Preliminary Results, in proceedings of the 27th International Cosmic Ray Conference (2003), p. 1849. 